\section{Demenz ausbremsen - Antihypertensiva reduzieren ist der falsche Weg}

\begin{abstract}
Hilft es, bei leichter Demenz die Antihypertensiva zu reduzieren, um das Gehirn auf Trab zu halten? Eine ungewöhnliche Studie zeigt eindeutig: Es bringt nichts.
\end{abstract}

$\mathrm{M}$ anche Krankheitskonzepte lassen sich nicht ausrotten, selbst wenn sie falsch sind. Wenn alte Menschen mit arterieller Hypertonie beginnen, „düddelig" zu werden, kommen sie oft auf den Gedanken, dass die blutdrucksenkenden Medikamente mit dafür verantwortlich sein könnten. Schließlich werde dem Gehirn mit der Blutdrucksenkung ja vielleicht etwas weggenommen, was es für eine geistige Leistungsfähigkeit benötige.

Dass dieses Konzept eines „kognitiven Bedarfshochdrucks" falsch ist, gilt unter Hypertonieexperten als unstrittig. Zwar war die Demenz in großen Hypertoniestudien nie ein primärer Endpunkt. „Wenn irgendetwas, dann waren Demenzen in den Behandlungsgruppen aber seltener als in den Kontrollgruppen", be- tonte Prof. Johannes Mann, Krankenhaus München-Schwabing. Seit Kurzem gibt es nun eine klinische Studie, die zusätzliches Futter liefert, wenn Patienten mit Verweis auf ihre Vergesslichkeit an der antihypertensiven Therapie zweifeln.

\section{Pillenpause ohne Einfluss auf's Hirn}

In dieser DANTE-Studie wurden 385 mindestens 75-jährige Patienten mit antihypertensiver Therapie und mildem kognitivem Defizit (MMSE 21-27 Punkte) rekrutiert. Die Patienten durften neben ihrer Hypertonie keine anderen schweren kardiovaskulären Erkrankungen oder entsprechende Ereignisse in ihrer Anamnese haben. Bei der Hälfte der Studienteilnehmer wurden die Antihypertensiva zu Studienzwecken randomi- siert abgesetzt. „Am Ende gab es weder bei der subjektiv empfundenen kognitiven Leistungsfähigkeit noch bei den Demenzparametern Unterschiede“, so Mann. Auch in den einzelnen kognitiven Domänen „Exekutivfunktionen“, "Gedächtnis" und „psychomotorische Geschwindigkeit" schnitten beide Gruppen nach 16 Wochen gleich ab, genauso wie bei Depressivität, funktionellem Status und Lebensqualität [1].

Der einzige Unterschied war wenig überraschend der Blutdruck, der in der Interventionsgruppe im Vergleich zur Kontrollgruppe um $7 / 2 \mathrm{mmHg}$ anstieg. Zwar sage diese Studie nichts über die langfristige Demenzentwicklung aus, so Mann. Darum gehe es Patienten, die ihre Medikamente absetzen oder reduzieren, aber auch gar nicht. Sie glauben vielmehr, dass sich ihre Vergesslichkeit kurzfristig bessert. Und das ist jetzt definitiv widerlegt. Phillip Grätzel

\section{Literatur}

1. Moonen J et al. JAMA Intern Med 2015; doi: 10.1001/jamainternmed.2015.4103

\section{Stärkere Blutdrucksenkung in der Schwangerschaft muss Kind nicht schaden}

\begin{abstract}
Die Blutdrucksenkung in der Schwangerschaft ist ein heikles Thema. In vielen Fällen können Ärzte zugunsten der Mutter aber etwas mutiger werden.
\end{abstract}

$\mathrm{D}$ as Grundproblem der Bluthochdrucktherapie von Schwangeren lässt sich nicht auflösen. Der Fetus kann schlecht damit umgehen, wenn die Plazenta therapiebedingt weniger durchblutet wird. Die Blutgefäße sind unreif. Eine Autoregulation findet intrauterin nur sehr begrenzt statt. Andererseits gefährdet Bluthochdruck in der Schwangerschaft die Gesundheit der Mutter.

\section{Leitlinien nehmen \\ kaum Druck von der Mutter}

Was also tun bei Frauen, die mit Bluthochdruck in die Schwangerschaft hineingehen? Medikamente müssen umgestellt werden, das ist klar. Zur Verfügung stehen im Wesentlichen Methyldopa, selektive Betablocker wie Labetalol, zudem
Nifedipin und Dihydralazin bzw. Hydralazin. In Sachen Blutdruckziele sind die meisten nationalen und internationalen Leitlinien sehr zurückhaltend. Eine Blutdrucksenkung aus mütterlicher Indikation wird meist erst ab $170 / 110 \mathrm{mmHg}$ empfohlen, bzw. ab 160/100 mmHg wenn der Bluthochdruck vorher bestand. Womöglich ist das etwas zu streng.

In einer randomisierten Studie aus Kanada wurde bei 1.000 Frauen in der 14. bis 34. Schwangerschaftswoche, die an einer vorbestehenden Hypertonie oder einem Gestationshypertonus ohne Proteinurie litten, eine strenge mit einer weniger strengen Blutdruckeinstellung mit einem diastolischen Zielblutdruck von 85 bzw. $100 \mathrm{mmHg}$ verglichen. Beim primären Endpunkt (fetale Komplikationen wie
Schwangerschaftsabbruch, Spontanabort, neonataler Tod) gab es keinen signifikanten Unterschied. Auch unterschieden sich die Kinder weder im Geburtsgewicht noch in ihrem Wachstumsverhalten. Seitens der Mutter traten schwere Komplikationen bei strengerer Einstellung hingegen numerisch seltener auf. „Insgesamt hilft uns die Studie, in der individualisierten Therapie zugunsten der Mutter etwas mutiger zu werden", kommentierte Prof. Duska Dragun, Charité Berlin. Zumindest wenn maternale Komplikationen aufträten, sei eine Blutdrucksenkung bis $85 \mathrm{mmHg}$ diastolisch erlaubt. (gvg)

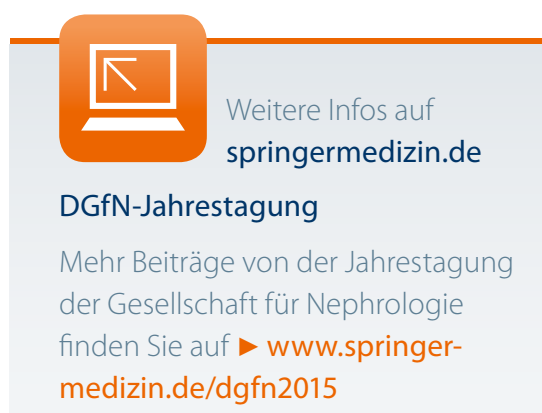

